\title{
Datability - Von Datensparsamkeit zu Datenfülle
}

\author{
Matthias Knoll • Knut Hildebrand
}

Eingegangen: 6. Oktober 2014 / Angenommen: 6. Oktober 2014 / Online publiziert: 6. Oktober 2014 (C) Springer Fachmedien Wiesbaden 2014

In nur wenigen anderen Bereichen der IT ist der Paradigmenwechsel so deutlich sichtbar wie im Bereich Datability. Dieses auf das Motto der CeBit 2014 zurückgehende Kunstwort umschreibt das, was uns alltäglich umgibt, die ,intelligente“ Analyse von vorhandenen Daten. Sie ermöglicht einerseits völlig neue Geschäftsmodelle, andererseits lassen sich Prozesse effizienter organisieren und Ressourcen optimieren.

Im positiven Sinn interpretiert erlaubt Datability den Unternehmen, sehr genau und vorausschauend $\mathrm{zu}$ agieren, dem Wettbewerb entsprechend angepasst $\mathrm{zu}$ entscheiden und die wirtschaftliche Entwicklung günstig zu beeinflussen. Datability sichert also langfristig Bestand und Fortentwicklung von Unternehmen und letztlich Arbeitsplätze. Probleme wie der Bullwhip-Effekt gehören damit hoffentlich ebenso der Vergangenheit an, wie strategische Fehlentscheidungen, etwa wenig nutzbringende Fusionen. Doch das alles hat seinen Preis. Nicht nur die Technologien und deren Einführung sind kostspielig - und meist langwierige, fachlich sehr anspruchsvolle Projekte. Auch die Datenqualität muss sehr hoch sein und das Personal exzellent qualifiziert, sonst ist der investierte Aufwand wenig wert. Im Extremfall kann sich alles ins Gegenteil verkehren. Erkenntnisse aus großen Datensammlungen ,fallen nicht vom Himmel“. Sie müssen hart erarbeitet werden!

Im negativen Sinn interpretiert befinden wir uns mitten in einem gesellschaftlichen Wandel. Denn Datability betrifft nicht nur Unternehmen, sondern uns alle. Überall hinterlassen wir mehr oder weniger breite Datenspuren, der NSA-Skandal,

\footnotetext{
M. Knoll ( $\square)$

Hochschule Darmstadt,

Darmstadt, Deutschland

E-Mail: matthias.knoll@h-da.de

K. Hildebrand

Hochschule Weihenstephan-Triesdorf,

Freising, Deutschland

E-Mail: knut.hildebrand@hswt.de
} 
massenhaft gestohlene Zugangsdaten und immer wieder eklatante Verstöße gegen den Datenschutz haben gezeigt, dass nicht nur innerbetriebliche Spezialinteressen im Vordergrund stehen, sondern viele „Player" an unseren Daten interessiert sind. Oft stellen wir fest, dass das Gut „Schutz der Daten“ global nicht überall gleich wertvoll ist. Bekannte Internet-Unternehmen nutzen unsere Daten als Rohstoff, um damit Geld zu verdienen. Das Paradoxon: Sind frühere Generationen für ihr Recht auf Datenschutz auf die Straße gegangen (Stichwort „Volkszählung“), so geben heute nicht nur die „Digital Natives“ ihre Daten mit erstaunlicher Gelassenheit und Bereitwilligkeit freiwillig an verschiedenste Organisation weiter. Manchmal bleibt uns nicht einmal eine Wahl, wollen wir am - digitalen - Leben teilhaben.

Oft wird argumentiert, „Ich habe doch nichts zu verbergen“. Das mag stimmen. Doch was, wenn Daten in falsche Zusammenhänge gestellt werden, weil Analysen fehlerhaft programmiert sind? Was, wenn die Ergebnisse überinterpretiert werden? Oder sich die eigene Meinung ändert, etwa im Kontext Berufseinstieg oder Familiengründung? - Das Internet vergisst nicht ...

Ohne in Panik auszubrechen ist es notwendig, sich über diese und andere Fragen im Kontext von Datability Gedanken zu machen und besonnen geeignete Maßnahmen auf privater, unternehmerischer oder staatlicher Ebene zu treffen.

Die beiden Beiträge zu diesem Paradigmenwechsel bieten hier Impulse. Der erste Beitrag von Peter Schaar, dem ehemaligen Bundesdatenschutzbeauftragten, befasst sich mit Datenschutz in Zeiten von Big Data. Der zweite Beitrag von Michael Mielke, Präsident der DGIQ, fordert Big Data als Chance zur Erhöhung der Datenqualität zu nutzen.

Knut Hildebrand und Matthias Knoll 